\begin{tabular}{|c|c|}
\hline Title & Theoretical Investigation of Phase Equilibria for Metal-Hydrogen A lloy \\
\hline Author(s) & Mohri, Tetsuo \\
\hline Citation & $\begin{array}{l}\text { Journal of Phase Equilibria and Diffusion, 28(1), } 72-78 \\
\text { https://doi.org/10.1007/s11669-006-9010-9 }\end{array}$ \\
\hline Issue Date & 2007-02 \\
\hline Doc URL & http:/hdl.handle.net/2115/28233 \\
\hline Rights & $\begin{array}{l}\text { Copyright @ 2007 A SM International. This paper was published in JOURNAL OF PHA SE EQUILIBRIA AND } \\
\text { DIFFUSION, 28, 1, 72 -78 and is made available as an electronic reprint with the permission of A SM International. One } \\
\text { print or electronic copy may be made for personal use only. Sy stematic or multiple reproduction, distribution to } \\
\text { multiple locations via electronic or other means, duplications of any material in this paper for a fee or for commercial } \\
\text { purposes, or modification of the content of this paper are prohibited. }\end{array}$ \\
\hline Type & article \\
\hline File Information & JPED28 1.pdf \\
\hline
\end{tabular}

Instructions for use 


\title{
Theoretical Investigation of Phase Equilibria for Metal-Hydrogen Alloy
}

Tetsuo Mohri

\author{
(Submitted October 27, 2006)
}

\begin{abstract}
Cluster variation method (CVM) was applied to calculate phase equilibria of metal-hydrogen systems. Two subjects are introduced in the present report. One is the summary of previous studies on the Pd-H system, and it is demonstrated that a single CVM free energy formula can systematically derive information of phase equilibria, intrinsic stability, and short range order diffuse intensities. The second subject is the theoretical calculations of superabundant vacancy (SAV) formation. Within the square approximation of the CVM, it is shown that abundant vacancies are introduced with the absorption of hydrogen when the interaction between vacancy and hydrogen is considered.
\end{abstract}

Keywords Cluster variation method, Pd-H phase diagram, Pd-H system, short range order diffuse intensity, square lattice, superabundant vacancy, tetrahedron-octahedron approximation

\section{Introduction}

The cluster variation method $(\mathrm{CVM})^{[1]}$ has been recognized as one of the most reliable theoretical tools in evaluating phase stability and phase equilibria. The topology of the phase boundary and the order of the transition derived and predicted by the CVM are quite accurate and satisfactory. Moreover, the wide range of atomic correlations explicitly considered in the free energy function provides detailed information of local atomic configurations in the equilibrium state. In fact, to identify the location of hydrogen in metalhydrogen systems is one of the most important subjects in clarifying both the stabilization and diffusion mechanisms, which aids to develop a new and efficient hydrogen storage material. The author has been attempting theoretical investigations $^{[2,3]}$ of the phase equilibria and phase stability of a metal-hydrogen system based on the CVM.

In the present study, summaries of the two theoretical investigations ${ }^{[2-4]}$ on metal-hydrogen systems performed by the author's group are provided. One is the calculations of

This article was presented at the Multi-Component Alloy Thermodynamics Symposium sponsored by the Alloy Phase Committee of the joint EMPMD/SMD of The Minerals, Metals, and Materials Society (TMS), held in San Antonio, Texas, March 12-16, 2006, to honor the 2006 William Hume-Rothery Award recipient, Professor W. Alan Oates of the University of Salford, UK. The symposium was organized by Y. Austin Chang of the University of Wisconsin, Madison, WI, Patrice Turchi of the Lawrence Livermore National Laboratory, Livermore, CA, and Rainer Schmid-Fetzer of the Technische Universitat Clausthal, Clauthal-Zellerfeld, Germany.

Tetsuo Mohri, Division of Materials Science and Engineering, Graduate School of Engineering, Hokkaido University, Sapporo 060-8628, Japan. Contact e-mail: tmohri@eng.hokudai.ac.jp

phase equilibria and site correlations of $H$ in $P d-H$ alloys. One peculiar feature of this system is that the phase separation takes place in the high temperature region while ordered phases appear in the low temperature region. Coexistence of these two opposite tendencies has attracted broad attention, and Oates and the present author ${ }^{[2,3]}$ attempted a series of theoretical investigations based on the phenomenological expression of the interaction energy combined with CVM. The major results are summarized in the first part of this report to demonstrate the reliability as well as a general framework of the CVM.

As a challenging topic of the applications of the CVM, theoretical calculations of superabundant vacancy (hereafter SAV) phenomena are attempted in the second part. It has been reported that due to the absorption of $\mathrm{H}$, an enormous amount of vacancies are introduced in metals and alloys. This phenomenon was first discovered by Fukai and his coworkers, ${ }^{[5]}$ and Fukai ${ }^{[6]}$ himself gave a clear explanation of the mechanism of the SAV in terms of the binding energy of vacancy and hydrogen. Also, Oates et al. attempted theoretical calculations ${ }^{[7]}$ by applying the Schottky-Wagner approximation to an appropriate set of species' chemical potentials. They further discussed the possibility of vacancy ordering at high vacancy concentration. However, these explanations were based on the Bragg-Williams approximation $^{[8]}$, which is a primitive point approximation in the CVM hierarchy and the atomic correlations among metallic atoms, hydrogen atoms, and vacancies are not properly taken into account. As far as the author's knowledge goes, no theoretical studies of SAV based on the CVM have yet been attempted. In the present report, preliminary calculations on a two-dimensional square lattice previously performed by the present author ${ }^{[4]}$ are presented.

\section{CVM Free Energy}

\subsection{Theoretical Methods}

Once an analytical expression of the free energy function is described, one can predict the equilibrium state from the 
vanishing condition of the first order derivative. This is a typical thermodynamics calculation of phase equilibria. It should be, however, noted that a reliable free energy function contains further information of stability of the system and local atomic configuration including site occupancies. The main objective of the present section is to provide examples of how a single CVM free energy function can yield systematic information concerning phase equilibria, stability, and site occupancies for the $\mathrm{Pd}-\mathrm{H}$ system.

In general, an alloy internal energy can be given in terms of cluster expansion ${ }^{[9]}$ written as

$E=\sum_{i}^{i_{\max }} v_{i} \cdot \xi_{i}$

where $i$ indicates various clusters which constitute the crystal, $v_{i}$ is the effective cluster interaction energy for $i$ cluster, and $\xi_{i}$ is the correlation function ${ }^{[10-12]}$ which is defined as the ensemble average of the spin operator $\sigma(p)$ which takes either +1 or -1 depending upon $A$ or $B$ atom, respectively, located at a lattice point $p$ in the $i$-cluster. The maximum cluster contained in the expansion is designated as $i_{\max }$ in $\mathrm{Eq} 1$, and the truncation of the expansion is closely related to the convergence of the interaction energies.

The location of $\mathrm{H}$ in $\mathrm{Pd}$ has been identified as the Octahedral site (hereafter abbreviated as $O$-site) in the face centered cubic lattice by neutron scattering experiment. ${ }^{[13,14]}$ Since the $O$-sites themselves form an fcc lattice, the phase equilibria for the Pd-H system can be treated by vacancy (hereafter Vac) $-\mathrm{H}$ binary equilibria on the fcc lattice immersed in the Pd lattice. Then, the entropy is calculated by directly applying the CVM of the fcc lattice. One of the essential conditions imposed on the free energy is that the maximum range of the interaction(s) assumed in the internal energy term designated by $i_{\max }$ should be contained in the largest cluster (basic cluster) in the entropy term. This is due to the fact that the range of atomic correlation generally exceeds those of atomic interactions. Since, as will be described latter, the internal energy given in the present analysis includes the second nearest neighbor pair interaction energy, the entropy term should include at least the second nearest neighbor pair correlations. The Tetrahedron-Octahedron approximation, ${ }^{[10,11]}$ therefore, is the minimum meaningful approximation and the entropy is given by

$$
S=k_{B} \cdot \ln \frac{\left(\prod_{i j k}\left(N \cdot z_{i j k}\right) !\right)^{8}\left(\prod_{i}\left(N \cdot x_{i}\right) !\right)}{\left(\prod_{i j k l m n}\left(N \cdot v_{i j k l m n}\right) !\right)\left(\prod_{i j k l}\left(N \cdot w_{i j k l}\right) !\right)^{2}\left(\prod_{i j}\left(N \cdot y_{i j}\right) !\right)^{6}}
$$

where $x_{i}, y_{i j}, z_{i j k}, w_{i j k l}$, and $v_{i j k l m n}$ are cluster probabilities of finding atomic arrangement specified by subscripts on a point, pair, triangle, tetrahedron, and octahedron clusters, respectively. In the conventional treatment, the numbers +1 and -1 are usually assigned to subscripts $i, j, k \ldots$ to represent $A$ and $B$ atoms, respectively. It should be noted that the entropy formula above is for a disordered phase and the one for an ordered phase can be derived by introducing sublattices.

It is important to note that the cluster probabilities, $\chi_{\{J\}}$, of atomic arrangement specified by $\{J\}$ is related to a set of correlation functions through a linear transformation,

$\chi_{\{J\}}=\frac{1}{2^{n}}\left\{1+\sum_{l \in J} V_{J, l} \cdot \xi_{l}\right\}$

where $n$ is the number of the lattice points constituting the cluster, and $V_{J, l}$ is termed $V$-matrix ${ }^{[10,11]}$, which is the sum of products of $i, j, k$, etc. The advantage of the correlation functions is the fact that they form an independent set of configurational variables while the cluster probabilities are mutually related through normalization and geometrical conditions.

Hence, the free energy of the system given by

$$
\begin{aligned}
F= & \sum_{i}^{i_{\max }} v_{i} \cdot \xi_{i}-T \cdot k_{B} \cdot \ln \\
& \frac{\left(\prod_{i j k}\left(N_{i j k}\right) !\right)^{8}\left(\prod_{i}\left(N \cdot x_{i}\right) !\right)}{\left(\prod_{i j k l m n}\left(N \cdot v_{i j k l m n}\right) !\right)\left(\prod_{i j k l}\left(N \cdot w_{i j k l}\right) !\right)^{2}\left(\prod_{i j}\left(N \cdot y_{i j}\right) !\right)^{6}}
\end{aligned}
$$

$=E\left(\left\{v_{i}\right\} ;\left\{\xi_{i}\right\}\right)-T \cdot S\left(x_{i}, y_{i j}, z_{i j k}, w_{i j k l}, v_{i j k l m n}\right)$

can be formerly rewritten as $F\left(\left\{v_{i}\right\}, T ;\left\{\xi_{i}\right\}\right)$ where $\left\{v_{i}\right\}$ and $T$ are given whereas $\left\{\xi_{i}\right\}$ are unknown quantities as variational parameters. Then, the equilibrium state is determined by minimizing the free energy $F$ with respect to correlation functions through

$\left.\frac{\partial F}{\partial\left\{\xi_{i}\right\}}\right|_{T,\left\{\xi_{j \neq i}\right\}}=0$

It is noted that $\left\{\xi_{i}\right\}$ at the equilibrium state provides information of local atomic configuration (short range order parameter) as well as site occupancies (long range order (LRO) parameter). Shown in Fig. 1 and Table 1 are the type of clusters considered in the Tetrahedron-Octahedron approximation for a disordered phase, and the correlation functions $\left\{\xi_{i}\right\}$ provide SRO parameters except the point correlation function $\xi_{1}$ which is linearly related to a concentration. When an ordered phase is considered, the number of correlation functions increases due to the breakdown of the symmetry of a disordered phase. In particular, the introduction of the sublattice induces more than a single point correlation function, $\xi_{1}^{\alpha}, \xi_{1}^{\beta} \ldots$ etc., and the LRO parameter can be defined by the difference of point correlation functions of different sublattices such as $\xi_{1}^{\alpha}-\xi_{1}^{\beta}$.

By considering the explicit site dependencies of the free energy, one can expand the free energy $F$ around the homogeneous state $F_{0}$. Then, the difference provides a 


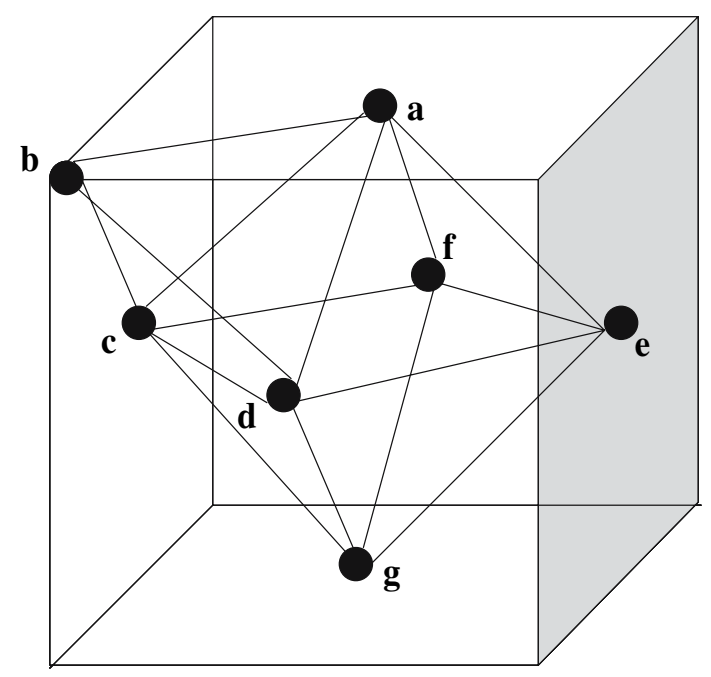

Fig. 1 Fcc lattice and a basic cluster employed in the Tetrahedron-Octahedron approximation of the cluster variation method (CVM)

Table 1 Clusters considered in the tetrahedron-octahedron approximation of the cluster variation method (CVM)

\begin{tabular}{lllllllllll}
\hline & $\xi_{1}$ & $\xi_{2,1}$ & $\xi_{3,1}$ & $\xi_{4,1}$ & $\xi_{2,2}$ & $\xi_{3,2}$ & $\xi_{4,2}$ & $\xi_{4,3}$ & $\xi_{5,1}$ & $\xi_{6,1}$ \\
\hline Lattice points & a & ab & acd & abcd & ag & ace & acde & cdef & acdeg & acdefg \\
a-g correspond to Fig. 1.
\end{tabular}

measure of the configurational fluctuation between two lattice points $p$ and $p^{\prime}$ which leads spatial inhomogeneity,

$\delta F=F-F_{0}=\frac{1}{2} \sum_{l, l^{\prime}} \sum_{p, p^{\prime}} F_{l l^{\prime}}\left(p, p^{\prime}\right) \delta \xi_{l} \cdot \delta \xi_{l^{\prime}}$

where $F_{l l^{\prime}}\left(p, p^{\prime}\right)$ is the second order derivative matrix defined as $\partial^{2} F /\left(\xi_{l}(p) \cdot \partial \xi_{l^{\prime}}\left(p^{\prime}\right)\right) \cdot{ }^{[15-18]}$ In fact, the first order derivative is a measure of the phase equilibrium while the second order derivative is that of the intrinsic stability of the system against fluctuation. The stability analysis is most conveniently performed by Fourier transforming $\delta F$ in Eq 7 into k-space:

$F_{t}\{\delta F\}=\frac{1}{2} \sum_{l, l^{\prime}} \sum_{\mathbf{k}} F_{l l^{\prime}}(\mathbf{k}) \cdot \delta X_{l}^{*}(\mathbf{k}) \cdot \delta X_{l^{\prime}}(\mathbf{k})$

$=\frac{1}{2} \sum_{l} \sum_{\mathbf{k}} \Lambda_{l}(\mathbf{k})\left|\delta Z_{l}()\right|^{2}$

where $F_{t}$ represents Fourier transformation, $\delta X_{l}^{*}(\mathbf{k})$ is the Fourier transformation of the deviation of correlation function, and the Hermitian properties of the second order derivative matrix is utilized to diagonalize the matrix in order to extract eigenvalues $\Lambda_{l}(\mathbf{k})$ in Eq 9. The physical meaning is interpreted as follows. If and only if all the eigenvalues are positive, the system is stable, while, as the temperature is lowered and one of the eigenvalues goes through 0 , the system becomes inherently unstable against the excitation and amplification of a particular wave vector $\mathbf{k}_{0}$ associated with the negative eigenvalue $\Lambda_{l}\left(\mathbf{k}_{0}\right)$. Then the limit of the stability of the system is given as the vanishing condition of the determinant of the second order derivative matrix, $\left|F_{l l^{\prime}}\left(\mathbf{k}_{\mathbf{0}}, c, T_{0}\right)\right|=0$, and the highest temperature $T_{0}$ at which this condition is satisfied is termed spinodal ordering temperature ${ }^{[17]}$ and the concentration wave associated with this negative eigenvalue is termed ordering wave. Moreover, the inverse of the second order derivative matrix is directly related to short range order diffuse scattering intensity at a specified point $\mathbf{k}$ as:

$\left.I_{\mathrm{SRO}}(\mathbf{k}, T) \propto k_{B} \cdot T \cdot F t\left\{\frac{\partial^{2} F}{\partial \xi_{j} \partial \xi_{k}}\right\}^{-1}\right|_{k=k *}$

Within the first and second nearest neighbor pair interaction energies for a fcc lattice, four dominant ordering waves are proposed. ${ }^{[16-18]}$ These are $\langle 100\rangle,\langle 11 / 20\rangle,\langle 1 / 21 / 21 / 2\rangle$, and $\left.\begin{array}{lll}0 & 0 & 0\end{array}\right\rangle$, and the wavelength and direction of each ordering wave reflects the underlying ordered phase. It was amply described that $\langle 100\rangle,\langle 11 / 20\rangle$, and $\langle 1 / 21 / 21 / 2\rangle$ waves yield $\mathrm{L}_{0}$, Chalocopyrite, and $\mathrm{L} 1_{1}$ ordered phases, respectively, and $\langle 000\rangle$ indicates the phase separation. It is, therefore, deemed useful to calculate the diffuse intensities for the four kinds of ordering waves to obtain information of a stable ordered phase in the low temperature.

\subsection{Phase Equilibria and Stability Analysis for Pd-H System}

Based on the measurements of relative standard hydrogen potential, Kuji et al. ${ }^{[19]}$ proposed that the internal energy $E(\theta, T)$ of Pd-H system can be tacitly given as the sum of two contributions:

$E=E_{\mathrm{C}}\left(\left\{\xi_{i}\right\}\right)+E_{\mathrm{NC}}(\theta, T)$

where $E_{\mathrm{C}}\left(\left\{\xi_{i}\right\}\right)$ and $E_{\mathrm{NC}}(\theta, T)$ are configuration dependent and independent contributions, respectively, and $\theta$ is the concentration defined as hydrogen/metal ratio which is equivalent to the point probability $x_{i}$ in Eq 2 to 4 . The configuration independent term $E_{\mathrm{NC}}(\theta, T)$ suggests a long range elastic interaction force induced by the absorption of $\mathrm{H}$ and, based on elasticity theory, this term is described ${ }^{[20]}$ in a polynomial expansion in powers of a point correlation function $\xi_{1}$ :

$E_{\mathrm{NC}}(\theta, T)=\sum_{i=0}^{i_{\max }} a_{i}(T) \cdot \xi_{1}^{i}$

Note $\xi_{1}$ is linearly related to the concentration, $\theta$, through $\theta=(1 / 2) \cdot\left(1+\xi_{1}\right)$, which is a particular expression of Eq 3 for a points cluster.

Then, by subtracting $E_{\mathrm{NC}}(\theta, T)$ from the experimentally obtained $E(\theta, T)$, the remaining configuration dependent energy $E_{\mathrm{C}}\left(\left\{\xi_{i}\right\}\right)$ is determined in the following form of cluster expansion given in Eq 1: 
$E_{\mathrm{C}}\left(\left\{\xi_{i}\right\}\right)=\sum_{i}^{i_{\max }} v_{i} \cdot \xi_{i}$

In a first principles calculations, $\left\{v_{i}\right\}$ are determined based on the electronic structure total energy calculations on a set of ordered compounds, while for the phenomenological schemes such as the present investigation, these are determined by trial-and-error schemes. The values determined $^{[2,3]}$ for the nearest $\left(v_{2,1}\right)$ and second nearest neighbor pairs $\left(v_{2,2}\right)$ and triangle $\left(v_{3}\right)$ interaction energies are $6.08 \times 10^{-3}(\mathrm{Ryd}), 7.06 \times 10^{-4}(\mathrm{Ryd})$ and $-2.84 \times 10^{-3}$ (Ryd), respectively.

A peculiar feature of the $\mathrm{Pd}-\mathrm{H}$ system is that the phase separation and ordering reaction coexist in the phase diagram. This is due to the interplay between $E_{\mathrm{C}}\left(\left\{\xi_{i}\right\}\right)$ and $E_{\mathrm{NC}}(\theta, T)$. The elastic interaction energy $E_{\mathrm{NC}}(\theta, T)$ inherently drives the system into phase separation. On the other hand, $E_{\mathrm{C}}\left(\left\{\xi_{i}\right\}\right)$ is the chemical interaction force confined in a few atomic distances and the positive value of $v_{2,1}$ indicates the ordering tendency. These two competing forces are the origins of the occurrence of the phase separation in the high temperature region and the ordering in the low temperature region. The present author and Oates ${ }^{[2,3]}$ successfully reproduced both the phase separation and ordering phase equilibria from a single free energy formula.

The diffuse intensity calculation $I_{\mathrm{SRO}}(\mathbf{k}, T)$ performed for the four kinds of ordering wave vectors, $\mathbf{k}$, in the previous study $^{[3]}$ suggests $I_{\mathrm{SRO}}(\mathbf{k}=1,1 / 2,0)>I_{\mathrm{SRO}}(\mathbf{k}=$ $1,0,0)>I_{\mathrm{SRO}}(\mathbf{k}=1 / 2,1 / 2,1 / 2)>I_{\mathrm{SRO}}(\mathbf{k}=0,0,0)$ for an entire temperature range $T$. In particular, $I_{\mathrm{SRO}}(\mathbf{k}=$ $1,1 / 2,0)$ is amplified as approaching some temperature $T^{*}=\sim 200 \mathrm{~K}$ with decreasing the temperature and all others decay, indicating that the system is unstable against the excitation and amplification of $\langle 11 / 20\rangle$ ordering wave which is propagated to form the Chalcopyrite ordered structure at low temperature. The intersection of the free energies (grand potential) of the disordered and Chalcopyrite phases at 50 at. $\%$ indicated ${ }^{[2]}$ that the ordering transition takes place at $T_{\mathrm{t}}=216 \mathrm{~K}$.

It is realized that the appearance of the Chalcopyrite structure is not trivial. Although the ground state analysis ${ }^{[21]}$ within the first and second nearest neighbor pair interaction energies predicted that the Chalcopyrite ordered phase is the most probable ordered phase with positive $v_{2,1}$ and $v_{2,2}$ described above, the existences of three body interaction energy $v_{3}$ and configuration independent energy $E_{\mathrm{NC}}(\theta, T)$ obscure the analysis. One notices that the short range order diffuse intensity calculation is quite powerful for predicting the possible ordered phase at low temperature. Another point is that $T^{*}$ is identified as the spinodal ordering temperature $T_{0}$. In view of the closeness of $T_{t}$ and $T_{0}$, weakly second order characters are expected for the disorder-Chalcopyrite transition.

The information in the correlation function $\left\{\xi_{i}\right\}$ for the equilibrium state can be employed ${ }^{[2]}$ to investigate the site occupancy of $\mathrm{H}$. By noting that a disordered phase is split into two sublattices, $\alpha$ and $\beta$, upon ordering of Chalcopyrite structure, temperature dependence of the LRO parameter $\eta=\xi_{1}^{\alpha}-\xi_{1}^{\beta}$ was calculated. ${ }^{[2]}$ The results indicate that $\mathrm{H}$ predominantly occupies the $\alpha$ sites in the low temperature region, while around $T=\sim 150 \mathrm{~K}, H$ starts to be transferred from $\alpha$ to $\beta$ sites, and at the transition temperature $T_{t}=216 \mathrm{~K}$ the distinction between two sublattices disappear. Similar calculations have been extended to multibody correlation functions and the results have already been reported elsewhere. ${ }^{[2]}$ In this manner, from a single free energy formula $F\left(\left\{v_{i}\right\}, T ;\left\{\xi_{i}\right\}\right)$ of the CVM, it is demonstrated that the first order derivative $\partial F / \partial \xi_{i}$ provides information of phase equilibria, the second order derivative $\partial^{2} F / \partial \xi_{i}^{2}$ can be employed to analyze the stability of the system against an excitation of a particular ordering wave, and that the equilibrium $\xi_{i}$ yields direct information of atomic configuration including site occupancies and site correlations.

\section{Superabundant Vacancies}

\subsection{Theoretical Descriptions}

In the calculations of Pd-H phase equilibria described in the previous section, what was considered is the Vac-H phase equilbria merely in the $O$-sites (interstitial site), which form fcc lattice. Superabundant vacancies, however, are induced by cooperative reactions of $M$ (Metallic atom), $H$ (hydrogen), and Vac (vacancy), and importantly Vac are introduced into the substitutional site (Metal site) with the absorption of $\mathrm{H}$ in the interstitial site. Hence, in order to fully account the configurational thermodynamics of SAV, it is necessary to construct a basic cluster, which contains both the substitutional and interstitial sites. It is realized that a square lattice shown in Fig. 2 contains both substitutional lattice sites $(\bigcirc)$ and $O$-site $(\square)$, and a square (shadowed) is regarded as the most primitive basic cluster which meets the above condition. Hence, we employed square approximation of the CVM.

The internal energy per a lattice point within the pair interactions can be written as

$E=\frac{Z_{1}}{2} \cdot \sum_{i j} \varepsilon_{i j}^{(1)} \cdot y_{i j}+\frac{Z_{2}}{2} \cdot \sum_{i j} \varepsilon_{i j}^{(2)} \cdot Y_{i j}$

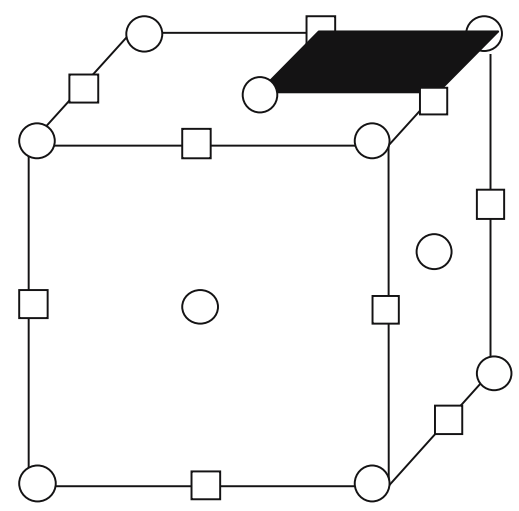

Fig. 2 Fcc lattice and a square lattice as a basic cluster of the square approximation of the cluster variation method (CVM). $\bigcirc$ (normal lattice site) and $\square$ (Octahedral interstitial site) are designated as $\alpha$ and $\beta$ sites, respectively 
where $Z_{1}\left(Z_{2}\right)$ is the number of the nearest neighbor (second nearest neighbor) lattice points, which is four (four) for the square lattice, $\varepsilon_{i j}^{(1)}\left(\varepsilon_{i j}^{(2)}\right)$ is the atomic pair interaction energy for the nearest neighbor (second nearest neighbor) pair, and $y_{i j}$ and $Y_{i j}$ are nearest and second nearest neighbor pair probabilities, respectively. For the sake of convenience, normal lattice sites and $O$-sites are designated as $\alpha$ and $\beta$, respectively, and $y_{i j}$ and $Y_{i j}$ are rewritten as $y_{i j}^{\alpha \beta}, Y_{i j}^{\alpha \alpha}$, and $Y_{i j}^{\beta \beta}$ in the following.

In order to assign the pair interaction energy, a LennardJones type potential is assumed,

$\varepsilon_{i j}(r)=\varepsilon_{i j}^{0}\left\{\left(\frac{r_{i j}}{r}\right)^{m}-2\left(\frac{r_{i j}}{r}\right)^{n}\right\}$

where $i$ and $j$ indicate metallic species (M), hydrogen $(\mathrm{H})$ and vacancy (Vac). In the present calculation, $m=8$ and $n=4$ are assigned as the exponent terms. The first term and second term indicate the repulsive and attractive potentials, respectively, and $\varepsilon_{i j}^{0}$ is a measure of the depth of the potential while $r_{i j}$ corresponds to the atomic distance at the bottom of the potential. The parameters employed in the present study are tabulated in Table 2. It is assumed that no interactions with $V a c$ exist. The smaller values of $r_{\mathrm{HH}}$ as compared with $r_{\mathrm{MM}}$ indicate the smaller size of hydrogen. By assigning stronger interaction between $\mathrm{M}$ and $\mathrm{H}$, one may expect to induce the alternative alignment of $M$ and $H$ on $\alpha$ and $\beta$ sites, respectively, which is the configuration of the metalhydrogen system.

At this point, the physical significance of the pair interaction energies assumed in the present calculations are discussed by referring to Fig. 3(a, b). In these figures, an open (solid) mark indicates that the lattice point is occupied by $M$ (for $\alpha$ site) and $H$ (for $\beta$ site) while the solid mark suggests a vacant (occupied by Vac) site. In Fig. 3(a), $\alpha$ sites

Table 2 Lennard-Jones parameters employed in the present calculation

\begin{tabular}{lccc}
\hline & H-H & H-M & M-M \\
\hline$e_{i j}^{0}$ & 1.0 & 2.0 & 1.0 \\
$r_{i j}$ & 1.0 & 1.1 & 1.2 \\
\hline
\end{tabular}

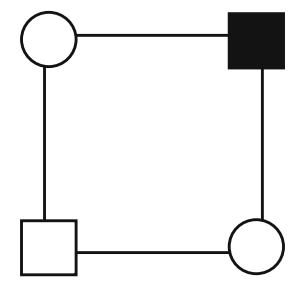

(a)

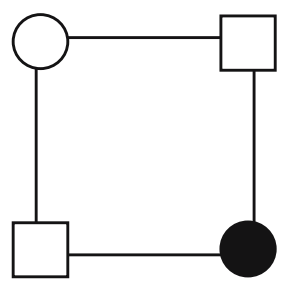

(b)
Fig. 3 Two typical configurations on the basic cluster. Open circle (square) indicates that the $\alpha(\beta)$ site is occupied by metal (hydrogen) while the solid mark indicates that the site is vacant. are occupied by $M$ and $\beta$ sites are by $H$ and Vac. Then, the pair interaction energies between $\alpha-\alpha$ and $\alpha-\beta$ reflect the cohesive energy of metal $M$ and dissolution energy of $\mathrm{H}$ in the matrix of M, respectively. In Fig. 3(b), one of $\alpha$ sites is occupied by Vac. In this case, the nearest neighbor pair interaction energy between $\alpha-\beta$ is the trapping energy of Vac by $\mathrm{H}$ which occupies $\beta$ site, while the second nearest neighbor pair interaction energy between $\alpha-\alpha$ reflects the formation energy of vacancy in the $M$ lattice.

It is recalled that phase equilibria for $\mathrm{Pd}-\mathrm{H}$ system in the previous section was reduced to vacancy (Vac) $-\mathrm{H}$ binary equilibria on the $\beta$ lattice immersed in the Pd atoms which fully occupy $\alpha$ sites. Hence, the nearest neighbor pair interaction $v_{2,1}$ discussed in the previous section corresponds to $\left(\varepsilon_{i i}^{(2)}+\varepsilon_{j j}^{(2)}\right) / 2-\varepsilon_{i j}^{(2)}$ across $\beta-\beta$ pair in the present square cluster. The positive $v_{2,1}$ determined in the previous section introduces Vac by enhancing the ordered configuration of $\mathrm{H}$ and Vac in the $\beta$ lattice, which is realized to be an unfavored initial configuration in the present simulation of SAV. The Lennard-Jones type potential given in Table 2 avoids such an unfavorable configuration by explicitly introducing the nearest neighbor (corresponding to one half the nearest neighbor of the fcc lattice) pair interaction with a deeper potential for $\mathrm{H}-\mathrm{M}$ pair. In fact, the energetics for binary $\mathrm{Pd}-\mathrm{H}(\mathrm{Vac}-\mathrm{H})$ equilibria introduced in the previous section is not sufficient to discuss the SAV, and the determination of realistic atomic interactions for ternary $\mathrm{M}-\mathrm{H}-\mathrm{Vac}$ remains as a future subject. The Lennard-Jones potentials introduced in the present study are regarded as preliminary potentials, which reveal minimum essential physics of the SAV.

The entropy is evaluated based on the square approximation of the CVM. ${ }^{[22-24]}$. The main task of the CVM is to assign the exponential term for each cluster probability. This is determined by several methods. In the present investigation, we employed Barker's procedure ${ }^{[25]}$ and the following expression was obtained:

$$
S=k_{\mathrm{B}} \cdot \ln \frac{\left(\prod_{i, j} N y_{i j}^{\alpha \beta} !\right)^{2}}{\left(\prod_{i} N x_{i}^{\alpha} !\right)^{1 / 2} \cdot\left(\prod_{i} N x_{i}^{\beta} !\right)^{1 / 2} \cdot\left(\prod_{i j k l} N w_{i j k l} !\right)}
$$

Then, the final expression of the free energy is given by combining Eqs 13 and 15 in the following way:

$$
\begin{aligned}
F & =E-T \cdot S \\
& =\frac{Z_{1}}{2} \cdot \sum_{i j} \varepsilon_{i j}^{(1)}(r) \cdot y_{i j}+\frac{Z_{2}}{2} \cdot \sum_{i j} \varepsilon_{i j}^{(2)}(r) \cdot Y_{i j} \\
& -T \cdot k_{B} \cdot \ln \frac{\left(\prod_{i, j} N y_{i j}^{\alpha \beta} !\right)^{2}}{\left(\prod_{i} N x_{i}^{\alpha} !\right)^{1 / 2} \cdot\left(\prod_{i} N x_{i}^{\beta} !\right)^{1 / 2} \cdot\left(\prod_{i j k l} N w_{i j k l} !\right)}
\end{aligned}
$$


It is noted that unlike the case of $\mathrm{Pd}-\mathrm{H}$ phase equilibria studied in the previous section, the determination of the phase equilibrium in the present section claims the minimization of $F$ not only with respect to cluster probabilities (correlation functions) but also with respect to $r$ :

$\left.\frac{\partial F}{\partial\left\{\xi_{i}\right\}}\right|_{T, r,\left\{\xi_{j \neq i}\right\}}=0$

and

$\frac{\partial E}{\partial r} \propto \frac{\partial E}{\partial V}=-p \cong 0$

In general, the indices $i$ and $j$ run from 1 to 3 corresponding to $\mathrm{M}, \mathrm{H}$, and Vac. Therefore, the vacancy concentration, $x_{3}^{\alpha}\left(x_{3}^{\beta}\right)$, which is one of the main concerns of the present study is obtained as the by-product of the minimization of $F$.

\section{Results}

Shown in Fig. 4 is the concentration of Vac as a function of the absorption (concentration) of $H$ up to $50 \%$ at $T=1.1$ where $T$ is the normalized temperature by $e_{11}^{0}$. The concentration of Vac nearly stays the same level for an entire range of concentration of $\mathrm{H}$. In fact, a slight amount of $\mathrm{Vac}$ shown in the figure under the absence of $\mathrm{Vac}-\mathrm{H}$ interactions is primarily due to the entropy effect. Although one should not forget the indirect interaction through the lattice expansion with the absorption of $\mathrm{H}$, which may

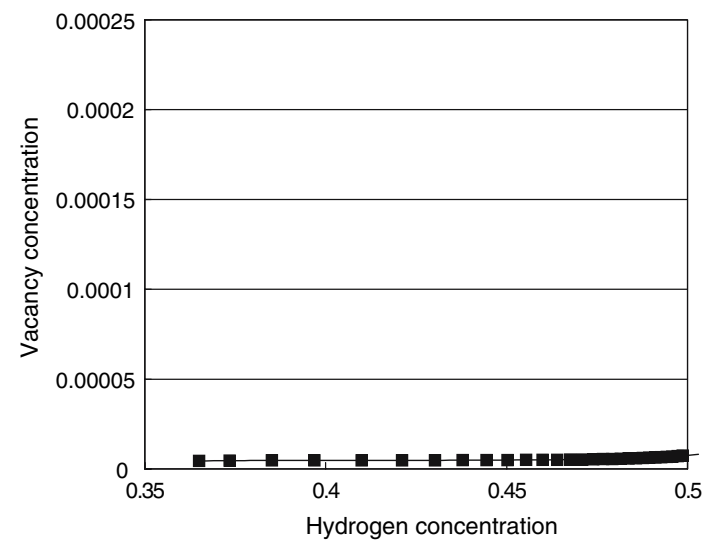

Fig. 4 Vacancy concentration as a function of hydrogen absorption when no interaction between hydrogen and vacancy is assumed

Table 3 Lennard-Jones parameters for the additional interaction between hydrogen and vacancy

\begin{tabular}{lll}
\hline & $\mathbf{C 1}$ & $\mathbf{C 2}$ \\
\hline$e_{\mathrm{HV}}^{0}$ & 0.5 & 1.2 \\
$r_{\mathrm{HV}}$ & 1.0 & 1.0 \\
\hline
\end{tabular}

enhance the formation of $\mathrm{Vac}$ with less energy expenditure, such an indirect interaction may induce more significant concentration dependences especially at the initial stage, i.e., in the lower concentration range of $\mathrm{H}$. We point out that the explicit consideration of long range elastic interactions through the expansion of matrix lattice due to the absorption of $\mathrm{H}$ and the contraction by the formation of Vac remain as one of the essential subjects in the future investigation.

Then, in order to investigate the trapping effect of Vac by $\mathrm{H}$ two kinds of Lennard-Jones type interaction energies are introduced between $\mathrm{H}$ and Vac. The Lennared-Jones parameters of two cases (Case 1 and Case 2 as abbreviated as $C 1$ and $C 2$, respectively) are tabulated in Table 3 in which one sees that $C 2$ is deeper than $C 1$. The resultant concentration of Vac calculated for each case is shown in Fig. 5 as a function of $\mathrm{H}$. One sees that the absorption of $\mathrm{H}$ induces the Vac in both cases. Furthermore, one confirms that the stronger the trapping is $(C 2)$, the higher the concentration of Vac becomes. This way, a preliminary calculation based on the CVM is able to confirm the essence of the SAV as the trapping effect of Vac by $\mathrm{H}$.

There are, however, number of points to be settled in the future investigations. One is that more realistic interaction energies among three kinds of species should be introduced by electronic structure calculations. The Lennard-Jones type potentials are oversimplified potentials for describing the interaction involving vacancies and atoms in the interstitial sites. Cluster expansion method, which was discussed in the previous section has been recognized as most powerful means to extract effective interaction energies. The selection of the super-cell structure for the metal hydrogen system including vacancies may be a challenging subject.

The second one is the local relaxation effects on the entropy calculations. Around the vacancy, one may expect a significant local relaxation effects, which alter the symmetry of the lattice. Hence the conventional square approximation, which assumes the equivalence of local and global symmetries, may not be justified. For this, continuous displacement cluster variation method $(\mathrm{CDCVM})^{[22-24,26]}$ should be addressed. The CDCVM was initiated to describe a free

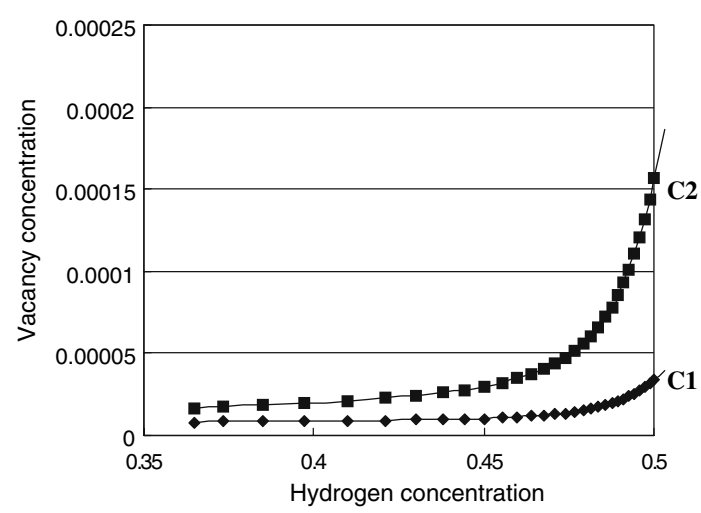

Fig. 5 Vacancy concentration as a function of hydrogen absorption for two kinds of interaction between hydrogen and vacancy. The Lennard-Jones parameters for $\mathrm{C} 1$ and $\mathrm{C} 2$ are tabulated in Table 3. 
energy of liquid, and the main attention has been recently turned towards the description of a topologically disordered structure of an alloy. The development of the CDCVM is still underway and full effort should be directed. Also, the two dimensional square lattice is the most primitive basic cluster and one needs a larger cluster in order to describe a realistic ordered phase such as the Chalcopyrite structure mentioned in the previous section. Finally, Vac and $\mathrm{H}$ are dealt with as conserved quantities in the present calculations. In reality, however, a system is open to both $\mathrm{H}$ and Vac and, therefore, one may not be able to map M-H-Vac equilibria onto a conventional ternary phase equilibria of a metallic alloy system. More rigorous studies are now undertaken and will be reported elsewhere.

\section{Acknowledgments}

The author acknowledges Professor Alan Oates for his kind guidance to the investigation of metal-hydrogen phase equilibria. Besides the studies of metal-hydrogen systems, the author enjoyed a lot of stimulating discussions of alloy phase equilibria with Professor Oates. The author is also grateful to Ms. Y. Jufuku for her aids of re-examining some of the calculated results through her BE thesis works. This work was supported in part by a Grant-in-Aid for Scientific Research on Priority Areas A of "New Protium Function" from the Ministry of Education, Science, Sport and Culture, of Japanese Government.

\section{References}

1. R. Kikuchi, A Theory of Cooperative Phenomena, Phys. Rev., 1951, 81, p 988

2. T. Mohri and W.A. Oates, Theoretical Investigation of Pd-H Phase Equilibria by the Cluster Variation Method, J. Alloys Compounds, 2002, 332-330, p 14

3. T. Mohri and W.A. Oates, CVM-based Calculation of the Pd-H Phase Diagram in the High Temperature Region, Mat. Trans., 2002, 43, p 2656

4. T. Mohri, Annual Report of Grant-in-Aid for Scientific Research on Priority Areas A of "New Protium Function" from the Ministry of Education, Science, Sport and Culture of Japanese Government, 2002, p 97, in Japanese

5. Y. Fukai, Y. Ishii, Y. Gotoand, and K. Watanabe, Formation of Superabundant Vacancies in Pd-H Alloys, J. Alloy Compounds, 2000, 313, p 121; Y. Fukai, T. Haraguchi, H. Shinomiya, and K. Mori, The Phase Diagram and Superabundant Vacancy Formation in Fe-H Alloys Under High Hydrogen Pressures, Scripta Materialia, 2002, 46, p 679; Y. Fukai and M. Mizutani, Phase Diagram and Superabundant Vacancy Formjation in Cr-H Alloys, Mat. Trans., 2002, 43, p 1079; Y. Shizuku, S. Yamamoto, and Y. Fukai, Phase Diagram of the Ni-H System at High Hydrogen Pressures, J. Alloys Com- pounds, 2002, 336, p 159; Y. Fukai, Y. Shizuku, and Y. Kurokaw, Superabundant Vacancy Formatin in Ni-H Alloys, J. Alloys Compounds, 2001, 329, p 195

6. Y. Fukai, Y. Ishii, Y. Goto, and K. Watanabe, Formation of Superabundant Vacancies in Pd-H Alloys, J. Alloy Compounds, 2000, 313, p 121

7. W.A. Oates and H. Wenzl, On the Formation and Ordering of ,Superabundant' Vacancies in Palladium Due to Hydrogen Absorption, Scripta Met. Mat., 1995, 33, p 185

8. W.L. Bragg and E.J. Williams, Proc. Roy. Soc., 1934, A145, p 69

9. J.W.D. Connolly and A.R. Williams, Density-Functional Theory Applied to Phase Transformations in Transition-Metal Alloys, Phys. Rev., 1983, B27, p 5169

10. J.M. Sanchez and D. de Fontaine, The fcc Ising Model in the Cluster Variation Approximation, Phys. Rev., 1978, B17, p 2926

11. T. Mohri, J.M. Sanchez, and D. de Fontaine, Binary Ordering Prototype Phase Diagrams in the Cluster Variation Approximation, Acta Metall., 1985, 33, p 1171

12. J.M. Sanchez, F. Ducastelle, and D. Gratias, Generalized Cluster Description of Multicomponent Systems, Physica (Utrecht), 1984, 128A, p 334.R

13. V.A. Somenkov and S.S. Shil'stein, Phase Tramsition of Hydrogen in Metals, Prog. Mat. Sci., 1980, 24, p 267

14. I.E. Worsham, M.K. Wilkinson, and C.G. Shull, J. Phys. Chem. Solids, 1957, 3, p 303

15. J.M. Sanchez, Pair Correlations in the Cluster Variation Approximation, Physica, 1982, 111A, p 200

16. T. Mohri, J.M. Sanchez, and D. de Fontaine, Short Range Order Diffuse Intensity Calculations in the Cluster Variation Method, Acta Metall., 1985, 33, p 1463

17. D. de Fontaine, k-Space Symmetry Rules for Order-Disorder Reactions, Acta metall., 1975, 23, p 553

18. D. de Fontaine, Ordering Instabilities and Pretransiton Effects, Metall. Trans., 1981, 12A, p 559

19. T. Kuji, W.A. Oates, B.S. Bowerman, and T.B. Flanagan, The Partial Excess Thermodynamic Properties of Hydrogen in Palladium, J. Phys. F: Met. Phys., 1983, 13, p 1785

20. R. Bass, W.A. Oates, H.R. Schober, and A. Stoneham, Configuration-Independent Elastic Interactions in MetalHydrogen Solutions, J. Phys., 1984, F14, p 2869

21. M.J. Richard and J.W. Cahn, Pairwise Interactions and the Ground state of Ordered Binary Alloys, Acta Metal., 1971, 19, p 1263

22. H. Uzawa and T. Mohri, Calculation of Short-Range-Order Diffuse Intensity for a Two Dimensional Square Lattice within Cluster Variation Method, Mat. Trans., 2001, 42, p 422

23. H. Uzawa and T. Mohri, Continuous Displacement Cluster Variation Method and Diffuse Scattering Intensity Calculation, Mat. Trans., 2001, 42, p 1866

24. H. Uzawa and T. Mohri, Continuous Displacement Cluster Variation Method in Fourier Space, Mat. Trans., 2002, 43, p 2185

25. A. Barker, Proc. Roy. Soc., 1953, A216

26. R. Kikuchi, Space is Continuous: Continuous Displacement Treatment of Phase-Separating Diagrams, J. Phase. Equilibria, 1998, 19, p 412 